\title{
RoboCup 2002 Small-Size League Review
}

\author{
Brett Browning \\ School of Computer Science \\ Carnegie Mellon University \\ Pittsburgh PA 15213, USA \\ brettb@cs.cmu.edu \\ http://www.cs.cmu.edu/ ${ }^{b r e t t b /}$
}

\section{Introduction}

The RoboCup challenge, to build soccer playing robots able to compete against the best human soccer players, is a goal well beyond our current levels of robot technology. Our current competition structure, with its various leagues, aims to step towards this goal by focusing on different aspects of the RoboCup problem. In the small-size league, global perception allows us to focus primarily on single and multi-robot control and multi-robot teamwork.

RoboCup 2002 showcased the significant level of progress made in the smallsize league over the last few years. Indeed, the small-size league has to be amongst the most exciting competitions at RoboCup. Significant advancements in robot design, control, and team coordination lead to the incredible 2002 small-size grand final between former two-time champions Big Red (Cornell University, US) and FU-Fighters (Free University of Berlin, Germany). Spectators and competitors alike, were truly in awe of the sheer speed of activity in the final game. Indeed, never in the history of RoboCup has a robot soccer final been played with such speed and control.

In many ways, RoboCup 2002 was a watershed year for the small-size league. We have made many changes to our competition, ranging from the qualification process through to challenge events and the way individual games are run, in order to push research developments within the competition to the next level. Some of the changes that we have begun will not be completed until RoboCup 2003 and beyond. We believe and hope that these changes will continue to make the small-size league a hotbed of robot intelligence development in the future.

In the following sections we will review the new changes that were wrought for the small-size league in 2002, as well as reviewing the main competition itself. We will finish our review of the small-size league with a projection of the changes we hope to finalize for RoboCup 2003 and where the future of this very exciting league is headed.

\section{New Features for 2002}

To reach the lofty goals of RoboCup, our competition must constantly evolve in synchronicity with the research developments by competition teams. If the competition fails to evolve, research developments will stagnate into over-engineered 
solutions that have no general applicability. For RoboCup 2002, we undertook a series of changes to our competition, some of which will not be complete until 2003 and beyond, that we hope will continue to push research developments in the coming years. In particular, our changes focused on the following goals:

1. Complete autonomy for the duration of the game

2. Large size field with no walls, with 11 vs 11 competitions

3. Regional competitions

4. Challenge events

5. FIFA compatible rules

6. Logging of each individual game for later analysis

Each of these changes has the goal of promoting research. Continuing the small-size tradition, the field was once again modified this year. A wider and longer field, along with a wider goal mouth, was used to help promote more interesting team game play. Our rule format has changed to be FIFA compliant, to help keep the competition in-line with the eventual goal of RoboCup. We have begun a process of moving towards using regional competitions, of which there is an ever growing number, to operate as qualification mechanisms for RoboCup. Using regional competitions in this way will help RoboCup grow beyond the constrained limitations of one event, and will continue to ensure that the RoboCup event maintains its status as the World Championships of robot soccer. To promote research in opponent modeling and associated opponentcustomized strategic planning, we have begun the first steps to log each and every game from a global perception perspective. These video logs will be available for teams to process after the competition. We hope that for RoboCup 2003, we will have on-line logging that teams will be able to access after each game of the competition.

\subsection{Challenge Events}

Perhaps the largest changes to the competition have been the introduction of Challenge events and the move to full autonomy for the entire duration of each game. Challenge events have been a regular item in the Sony legged league and Simulator leagues for a while now. Essentially, they promote quantitative evaluations of particular aspects of the robot technology that is developed to play robot soccer. In Fukuoka, we introduced three challenges for the first small-size Challenge competition. The three challenges range in difficulty and are designed to quantitatively compare each team as well as to challenge the state-of-the-art. The first two challenges fit well within any team's capabilities, while the third challenges the abilities of every team. The three challenges are:

- Challenge 1: The navigation challenge. One robot must navigate from defense box to defense box through an unknown, static, robot-sized obstacle field. The robot must complete five laps, where each lap consists of traveling from the start defense box to the opposite defense box and back. A penalties of $10 \mathrm{~s}$ is added for each collision, no matter how small, between the robot and the obstacles. 
- Challenge 2: The shooting challenge. One robot must score as many goals as possible within 2 minutes. Each time the robot scores, a ball is released on the halfway line on top of the 45 degree sidewall causing the ball to roll into the middle of the field with some variation. At the same time the scoring ball is removed from the field.

- Challenge 3: The passing challenge. Two robots, each restricted to one half of the field, must pass the ball back and forth. Each complete alternate pass is counted. An alternate pass is one where robot A passes to robot B and then robot $\mathrm{B}$ passes back to $\mathrm{A}$ on the opposite side of the circle from the original pass. As with challenge 2, the robots have 2 minutes to complete as many alternate passes as possible.

As expected, the first two challenges were easily completed by all teams while challenge three proved difficult for all but except Cornell Big Red (indeed most teams did not participate). RoboDragons (Aichi Prefectural University, Japan) in their first RoboCup competition were the surprise team by defeating defending champions LuckyStar (Ngee Ann Polytechnic, Singapore) in the goal scoring challenge. The final results for the top four placings were:

\begin{tabular}{||c||ll|ll|ll||}
\hline \hline Rank & Challenge 1 & & Challenge 2 & \multicolumn{4}{|l||}{ Challenge 3 } & \\
\hline \hline 1 & Luckystar & 24.9 & RoboDragons & 23 & Cornell & 10 \\
\hline 2 & FUFighters & 27.2 & LuckyStar & 22 & Roobots & 1 \\
\hline 3 & Cornell & 28.8 & Fufighters & 19 & CMDragons02 & $1 / 2$ \\
\hline 4 & CMDragons02 & $28.7+1$ hit & Roobots & 14 & & \\
\hline \hline
\end{tabular}

\subsection{Complete Full Game Autonomy}

While our robot systems are autonomous, until this competition, all the referee commands were translated to the robot teams via human operators from each development team. Previously, robots were physically moved into game restart positions such as free kicks, penalties, and kick offs, and system autonomy was started and stopped via computer commands activated by a human operator from each team (ie. robots would be halted during game stoppages). Such a game structure often lead to boring sections where a human would take a significant amount of time to micro-position a robot to take a kick, often to no great effect. Secondly, teams could gain milliseconds of advantage by guessing their system latency to activate their system earlier than the referee's whistle thereby guaranteeing their robots would move first. Given the powerful kicking mechanisms that some robots possess, starting milliseconds ahead of the other team can make a substantial difference in the state of the game. Finally, RoboCup is aimed towards developing intelligent autonomous robots. Thus, having completely autonomous robots for the entire duration of each game is in-line with the goals of RoboCup.. 
To address these concerns, the organization committee for 2002 developed the Referee Box. In essence, the referee box translates the referee's directives into computer usable signals thereby removing the human operators from the loop. Although the current referee box implementation translates all the referee's commands, only start and stop commands were compulsory for 2002. Nearly all the teams were able to start and stop autonomously. Some teams, were able to operate completely autonomously for nearly the entire game (eg. CMDragons02 (Carnegie Mellon, USA)), which certainly made the robot handler's job easier! For teams that could not operate autonomously with the start and stop signals, a penalty was incurred. A neutral human operator was required to restart the penalized team at the referee's command thereby incurring significant restart latency. For RoboCup 2003, the small-size league will move to complete autonomy for the duration of the game. Thus, no human operator will be allowed to touch a robot or computer while the game is in progress except during time-outs and half-time breaks.

The current implementation of the referee box is a computer program with a Graphical User Interface (GUI) that runs on a laptop. The laptop is connected to each team computer via a spilt RS-232 serial cable running at 9600bps with no parity, 8 data bits, and 1 stop bit. The assistant referee, who previously had time-keeping duties, operates the referee box by clicking on the appropriate button in accordance with the referee's commands.

The full details of the 2002 referee box implementation are available online at http://www.cs.cmu.edu/ ${ }^{\sim}$ brettb/robocup/referee.html. The referee box is available for operation in both Microsoft Windows environments, and Linux environments. The source code is freely available in both cases. Each program maintains a running clock, the game score, the time-out clocks and available time-outs, and translates each referee command into a single ASCII character that is sent to each team via the serial cable. The protocol is simple to ensure that all teams are able to use it properly, although we expect to extend and modify the protocol when required. Currently, the referee box does not log any information, although we expect to include this operation in the next release.

On the whole, the referee box was a wonderful success. One cannot imagine how the final, where both teams were capable of very high accelerations, would have been manageable without its use. We hope that for RoboCup 2003, no team will require human intervention during the game.

\section{Main Competition}

The main competition demonstrated clear advancements in the level of technology. Clearly, the leading teams have progressed a long way. More interestingly, the main body of the competition has steadily improved as well. Most teams had working robots capable of playing a game of soccer that would be competitive at previous RoboCups. Indeed, the improvements within the league were evident early on during the qualification process.

Teams were required to submit their team description paper and video footage of their team playing soccer as part of the qualification process. The or- 
ganization committee ranked the teams, based in part on the video footage and prior team performance, and selected the top 20 teams for the main competition. Due to the limitations on time and space, only 20 teams could be accommodated at RoboCup. Thus, from the 31 teams that submitted the qualification material, only 20 could participate. We hope that in the future regional competitions will take the place of the qualification process, and will also provide an avenue for RoboCup to grow beyond the finite limitations of the one event. As part of the qualification process, the top eight teams were given a ranking based on prior performance and allocated to one of the four groups. The remaining teams were allocated to groups randomly. Within each group, a round-robin tournament was held during the first three days of competition with the top two teams from each group entering the elimination rounds.

There were a number of surprises during the round-robin phase. The RoboDragons, a first-time RoboCup team, followed up on their challenge winning performance by reaching the quarter finals. Along the way they defeated the RoboRoos (University of Queensland, Australia) in a very close game. They met their match in the quarter final, however, in the form of the FU-Fighters. The FU-Fighters introduced specially designed omnidirectional wheels that enabled their robots to accelerate at incredible speeds. Exact figures were not available, but accelerations figures of close to $5 \mathrm{~m} . \mathrm{s}^{-1}$ would not be surprising. Big Red, demonstrated a similar high-speed approach where four wheels were the key to acceleration rather than special wheels. Big Red also unveiled another innovation: a super high-speed dribbler. With their new dribbler, the Big Red robots were able to drag the ball around the field at will. This tactic, a turnaround from their passing approach in 2001, formed a significant part of their game strategy that produced results. CMDragons02, another quarter final team, demonstrated a unique software innovation: an autonomous play-based strategy engine that adapted on-line to the weaknesses of the opponent. Although Carnegie Mellon has previously demonstrated adaptive role-switching and on-line planning [1, to the author's knowledge this is the first use of adaptive strategy in any of the real-robot leagues.

The full list of quarter finalists includes LuckyStar, Field Rangers (Singapore Polytechnic, Singapore), FU-Fighters, RoboDragons, Roobots (University of Melbourne, Australia), CMDragons'02 (Carnegie Mellon, USA), Big Red, and newcomers IUT Flash (Isfahan University of Technology, Iran). Of these teams, Big Red, FU-Fighters, LuckyStar, and Roobots, went on to the semi-finals. Here, defending champions LuckyStar came up short in the game of speed against FUFighters. It now seems that the FU-Fighters have become the informal speedkings of RoboCup and heir apparent to LuckStar. LuckyStar went on to beat Roobots, who lost to BigRed in the other semi-final, for third.

The grand-final between FU-Fighters and eventual champions Big Red, proved to be the climactic event of the competition. These two teams have played on numerous occasions in previous competitions dating back to RoboCup'99. Both teams have very aggressive strategies. The resulting game was a spectators delight. Big Red used their fast acceleration and powerful dribbler to move the 
ball around the field. Meanwhile FU-Fighters used their incredible speed and chip-kicker to attack, attack, attack. In two 10 minute halves no less than 10 goals were scored. Indeed, the first two goals (the first to Big Red, the second to FU-Fighters) came within the first minute of the game, which is certainly a small-size grand-final record. Once the dust had settled, Big Red had reasserted their dominance in the small-size league winning for the third time and it was once again the end of another exciting RoboCup competition. One wonders what new wonders will be in store for RoboCup 2003.

For interested readers, the following web-links are relevant:

- RoboCup 2002 scores: http://www.robocup2002.org.

- Small-size 2002 web-site: http://www.cs.cmu.edu/ ${ }^{\sim}$ brettb/robocup.

- RoboCup main web-site: http://www.robocup.org/.

\section{Summary of 2002, the Future and Results}

In these few pages, we have tried to highlight many of the competition changes, and technological innovations that made RoboCup 2002 small-size such a wonderful competition. This is an ever-evolving competition where cutting edge research is being performed and that research is evaluated in the hardest possible way. The most notable achievements, research-wise, are the movements to full autonomy and the advances in high-speed control and adaptable strategy.

The stage is now set for RoboCup 2003 to be a very exciting year. The changes that have been wrought in the competition will continue to evolve. In particular, RoboCup 2003 will present the first robot league with autonomy for the entire game. Additionally, much work is in progress to broaden the research impact of the league. Collaborative efforts between teams are currently underway to develop and release an open source simulator, and an open source vision system. Likewise, efforts are currently underway in some camps to completely release RoboCup 2002 source code. Such open source approaches will further improve the research impact of our competition and help reduce the start up work required by new teams. The future for small-size is bright indeed.

\section{References}

1. Veloso, M., Bowling, M., Achim, S., Han, K., and Stone, P., "The CMUnited-98 champion small robot team", In Asada, M., and Kitano, H., (eds), RoboCup-98: Robot Soccer World Cup II, pages 77-92. Springer Verlag, Berlin, 1999. 


\section{Appendix: Results}

Table 1. Qualified f-180 Small Size Teams

\begin{tabular}{|l|l|l|}
\hline Lucky Star & Ngee Ann Polytechnic & Singapore \\
5DPO & Universidade do Porto & Portugal \\
All Botz & University of Auckland & New Zealand \\
CM Dragons 02 & Carnegie Mellon University & USA \\
Team Canuck & University of Alberta & Canada \\
Cornell Big Red & Cornell University & USA \\
FU-Fighters & Freie Universität Berlin & Germany \\
Field Rangers & Singapore Polytechnic & Singapore \\
IUB Team 2002 & International University Bremen & Germany \\
IUT Flash & Isfahan University of Technology & Iran \\
KU-Boxes 2002 & Kinki University & Japan \\
OMNI & Osaka University & Japan \\
Owaribito-CU & Chubu University & Japan \\
RobBobcats & Ohio University & USA \\
RoboDragon & Aichi Prefectural University & Japan \\
RoboRoos & University of Queensland & Australia \\
ROGI Team & Universidad de Girona & Spain \\
Roobots & University of Melbourne & Australia \\
Sharif CESR & Sharif University of Technology & Iran \\
RoboSix Team & University Pierre and Marie Curie & France \\
\hline
\end{tabular}

Table 2. Results: f-180 Small Size League

\begin{tabular}{|l|l|l|l|}
\hline 1 & Big Red & Cornell University & USA \\
2 & FU-Fighters & Free University of Berlin & Germany \\
3 & Lucky Star & Ngee Ann Polytechnic & Singapore \\
\hline
\end{tabular}

